\title{
Mini review on Keto-Enol ratio of curcuminoids
}

\author{
Sameera A. Rege, Megha Arya, Shamim A. Momin
}

\author{
Institute of Chemical Technology, Matunga (East), Mumbai, India
}

\author{
Keywords: \\ Curcuminoid \\ Keto-enol \\ Methoxy
}

\section{Article history:}

Received

20.01.2019

Received in

revised form

29.04.2019

Accepted

30.05.2019

\section{Corresponding author:}

Rege A. Sameera

E-mail:

sameerarege@

gmail.com

\section{DOI:}

$10.24263 / 2310-$

$1008-2019-7-1-5$

\section{Abstract}

Introduction. Curcumin, demethoxycurcumin and bisdemethoxycurcumin collectively constitute curcuminoids, exhibit different rates of degradation as well as therapeutic properties. Their remarkable dissimilar behaviour is affiliated to their tendency to undergo keto-enol tautomerism whereas their rates of degrading and imparting therapeutic properties depend on the ratio of keto and enol tautomers of individual curcuminoid.

Materials and Methods. The aim of the current review is to focus on the ratio of keto and enol tautomers and to explain how the ratio of the two tautomers affects the rate of activities of the curcuminoids. The papers regarding variations in the rate of activities of curcuminoids were scrutinized. The factors affecting the ratio of keto-enol tautomerism were studied.

Results and discussion. Structurally, curcuminoids are the compounds possessing $\beta$-diketone moiety. The characteristic property of compounds containing $\beta$-diketone moiety is to exhibit keto-enol tautomerism. Hence, each curcuminoid is a mixture of keto and enol tautomers. The keto tautomer of curcumioids is responsible for their therapeutic activities. On the other hand, enol tautomer of curcuminods has tendency to undergo degradation. Hence, the ratio of keto and enol tautomer needs to be considered while studying the activities of curcuminoids.

Nevertheless, the ratio of keto and enol tautomer depends on the existence of other functional groups. Because of the presence of electron donating hydroxyl group, the proportion of enol tautomer predominates in curcuminoids. Further, due to the presence of two more electron donating methoxy groups in curcumin, the ratio of the enol tautomer is higher and hence, the susceptibility to undergo degradation is greater than demethoxycurcumin, which contains only one methoxy group. The ratio of the enol tautomer is minimum in bisdemethoxycurcumin amongst all the curcuminoids, which is devoid of methoxy group. Consequently, bisdemethoxycurcumin has the least rate of degradation amongst curcuminoids. Methoxy group enhances the activity of the functional sites in curcuminoids in keto form. Therefore, curcumin shows maximum beneficial activities in keto form amongst all the curcuminoids, followed by demethoxycurcumin and bisdemthoxycurcumin. Thus, the rate of the activities displayed by curcuminoids is governed by their keto and enol proportion.

Conclusion. It can be concluded from this review article that besides $\beta$-diketone moiety, responsible for keto-enol tautomerism, other groups also play an important role in determining the ratio of keto and enol tautomers. 


\section{Introduction}

Turmeric (Curcuma longa L.) is one of the herbs, which is extensively used as a spice. It is mainly cultivated for its rhizome. Curcumin, demethoxycurcumin and bisdemethoxycurcumin constitute curcuminoids, which are the polyphenolic compounds present in the turmeric rhizome [1]. The composition of curcuminoids was found to be curcumin 60-80\%, demethoxycurcumin 15-30\%, bisdemethoxycurcumin 2-6\% [2]. Curcuminoids are the natural colouring agents used in food industry. The three curcuminoids are proven to have various therapeutic properties such as antioxidant [3], anti-inflammatory, wound healing $[4,5]$, anticancer $[6,7]$ and anti-depressant $[8,9]$. They are used to prevent Alzheimer's disease [10].

However, the overall activity of the mixture of curcuminoids is attributed to the three individual curcuminoids and each shows a different rate of activity [10]. The three curcuminoids display different rates of degradation as well [11]. In this review, our main focus is to evaluate the root cause of the different rates of activities of each curcuminoid.

\section{Materials and methods}

Phenolic hydroxyl groups and the heptadienone moiety are responsible for the beneficial activities of curcuminoids. The dienone moiety is accountable for keto-enol tautomerism. The enol tautomer is liable to degradation. The difference in the keto-enol ratio of each curcuminoid is the key parameter for the different rates of beneficial activities as well as degradation shown by curcuminoids. The ratio of keto-enol tautomers is influenced by the other groups present on phenolic rings. The objective of our study is to show how the overall structure of each curcuminoid contributes to the keto-enol ratio and hence, the rate of activity.

Review is constructed on the basis of previously available research articles.

\section{Results and discussion}

\section{Proportion of keto and enol tautomers of curcuminoids}

The curcuminoids exhibit keto-enol tautomerism, structures (1) and (2) (Figure 1), a typical property of the compound having $\beta$-diketone moiety [12]. The enol tautomer is certainly more stable than keto tautomer due to resonance of conjugated pi system and intramolecular hydrogen bonding [13]. Also, since they possess electron donating hydroxyl group in the aromatic ring and electron withdrawing carbonyl group in heptadienone moiety, structures (3) and (4) can be drawn respectively. The existence of structures (3) and (4) has already been proven by NMR data [14]. Structures 3 and 4 resemble the enol tautomer (Figure $1)$. 


\section{- Food Technology}

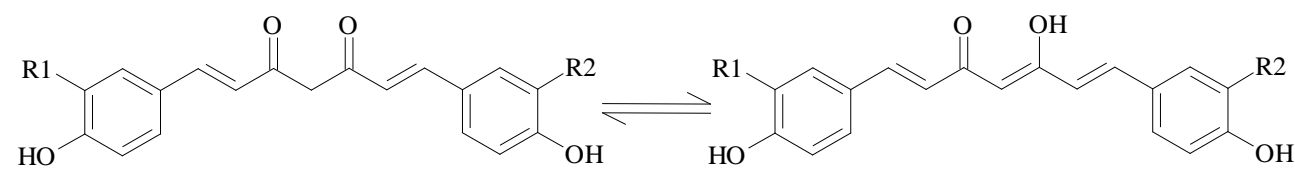

1

Keto

2

Enol

(i) Tautomerism due to $\beta$-diketone moiety

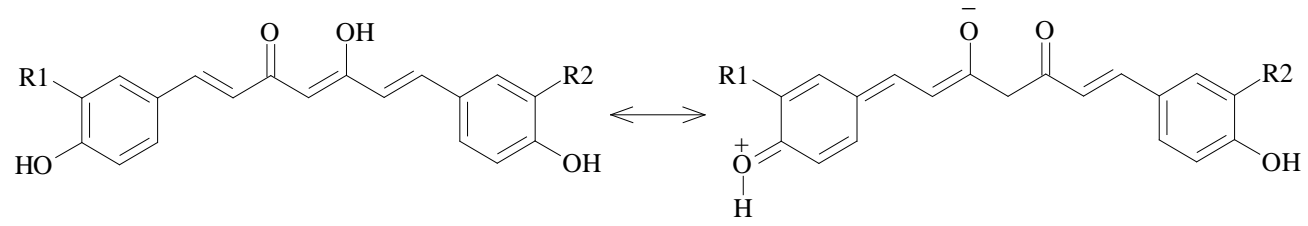

2

Enol

3

Zwitterion

(ii) Formation of zwitterion (3) due to hydroxyl group

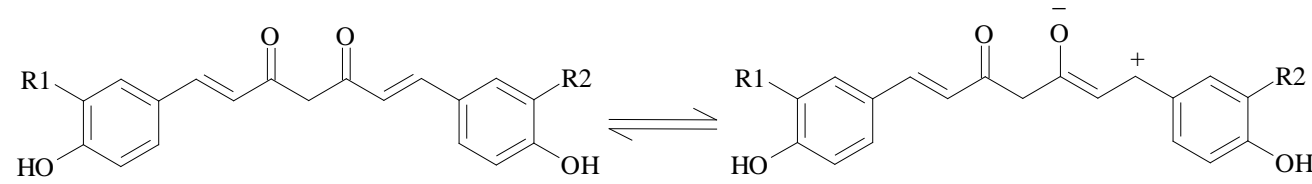

1

Keto

4

Zwitterion

(iii) Formation of zwitterion (4) due to carbonyl group

Figure 1. Tautomerism of curcuminoids:

(i) $\mathrm{R} 1=\mathrm{R} 2=-\mathrm{OCH}_{3}$; Curcumin

(ii) $\mathrm{R} 1=-\mathrm{OCH}_{3}, \mathrm{R} 2=-\mathrm{H}$; Demethoxycurcumin

(iii) $\mathrm{R} 1=\mathrm{R} 2=-\mathrm{H}$; Bisdemethoxycurcumin

Thus, it can be represented as,

Keto $\rightleftharpoons$ Enol

Moreover, due to the presence of two additional electron donating methoxy groups in curcumin, the proportion of enol tautomer is maximum amongst all the curcuminoids. In case of demethoxycurcumin, which contains only one additional electron donating methoxy 
group, the proportion of enol tautomer is higher compared to bisdemethoxycurcumin which is devoid of additional electron donating group. Thus, the different ratios of keto and enol tautomers of the curcuminoids are dependent on the number of the methoxy groups present. Even though the electron donating group prefers the enol tautomer, it does not shift the equilibrium completely to the enol form [15].

Based on the above discussions, we are proposing the following resonating structures of demethoxycurcumin and curcumin (Figure 2).

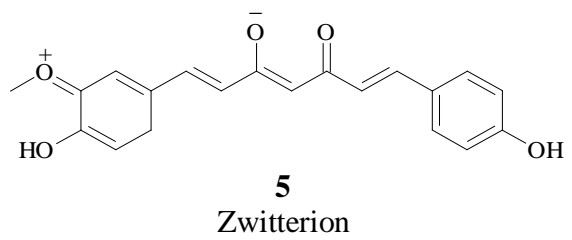

(i) Formation of zwitterion (5) in demethoxycurcumin

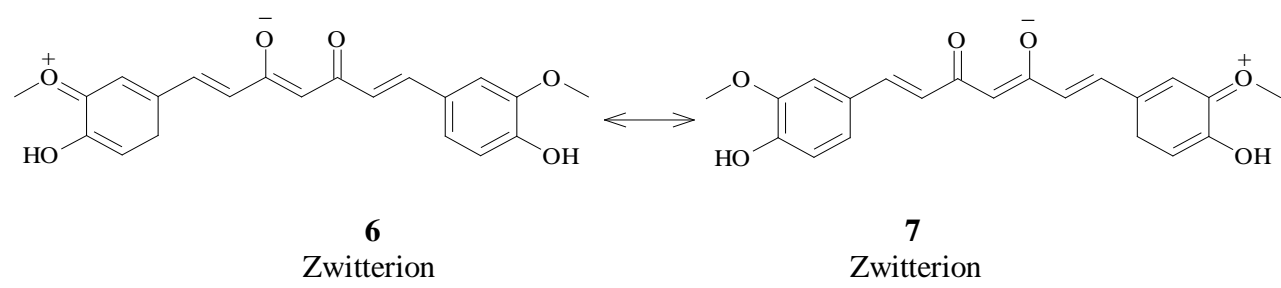

(ii) Formation of zwitterions (6) and (7) in curcumin

Figure 2. Formation of zwitterions due to methoxy groups

Because of the presence of a single methoxy group in demethoxycurcumin, an additional resonating structure (5) is formed, which resembles the enol tautomer. Hence, the proportion of enolic form of demethoxycurcumin is higher than bisdemethoxycurcumin. Similar explanation can be given for the maximum enolic content of curcumin amongst curcuminoids, which contains two methoxy groups thereby forming two additional resonating structures (6 and 7).

\section{Effect of different ratios of keto-enol tautomers of curcuminoids on their individual rate of activity}

Under alkaline and non-polar medium, since the enol tautomer of curcuminoids undergoes degradation [16, 17], curcumin degrades at the highest rate followed by demethoxycurcumin and bisdemethoxycurcumin. The beneficial effects of all the curcuminoids can only be achieved when they do not undergo degradation, i.e., when the activity of keto tautomer predominates that of enol in acidic or polar medium. Though the proportion of keto tautomer is same in all the three curcuminoids, they differ in the rate of 
activity depending on the number of methoxy groups present in them. Methoxy group, being an electron donating group, increases electron density on hydroxyl groups and diketone moiety, which are the functional sites of the curcuminoids [18]. Hence, the rate of activities associated with hydroxyl groups and diketone moiety also enhances due to presence of methoxy group. As curcumin has two methoxy groups, the rate of activities linked with hydroxyl group and diketone moiety is higher than demethoxycurcumin, which contains only one methoxy group. Bisdemethoxycurcumin does not contain a methoxy group, hence shows the least rate of activities amongst all the curcuminoids.

\section{Exclusive activity of curcuminoids as keto or enol tautomer depending on the medium}

Both, keto and enol tautomers of curcuminoids are present in varied ratio in any medium. Nevertheless, the equilibrium ratio of the two tautomers in a medium is constant. The activity of one tautomer leads the other depending on the polarity of the medium [19]. If any one of the keto or enol tautomers is removed, more of the same tautomer will be formed to retain the equilibrium. Hence, in an acidic or polar medium, when the activity of keto tautomer of any of the curcuminoids predominates, the keto tautomer gets utilized thereby decreasing its quantity; the enol tautomer gets converted to the keto tautomer to maintain the equilibrium. Hence, the molecule as a whole displays the activity of keto tautomer.

Likewise, in basic or non-polar medium, when the enol tautomer takes up the activity, the keto tautomer gets converted to enol tautomer and the molecule gets degraded.

\section{Conclusion}

The two factors that influence the activity of curcuminoids are medium and structure. The mode of activity of curcuminoids (viz. keto or enol) is determined by the nature of the medium while the rate of activity of curcuminoids is determined by the structure. Though the $\beta$-diketone moiety present in the three curcuminoids is responsible for keto-enol tautomerism of the curcuminoids, the equilibrium shift towards enol tautomer depends on the other groups present in the molecule. Hence, the variation in the amount of keto and enol tautomers of the three curcuminoids is observed. The rate of activity of keto tautomer and the proportion of enol tautomer are directly dependent on the number of methoxy groups present. The number of methoxy groups is in the order of curcumin > demethoxycurcumin > bisdemethoxycurcumin. Hence, the rate of valuable activities of keto tautomer and degradation of enol tautomer is also in the same order. Further research needs to be carried out to find out the exact ratio of keto and enol tautomers of curcuminoids.

\section{References}

1. Jayaprakasha G.K., Rao L.J, Sakariah K.K. (2005), Chemistry and biological activities of Curcuma longa, Trends in Food Science and Technology, 16(12), pp. 533-548.

2. Wichitnithad W., Jongaroonngamsang N., Pummangura S., Rojsitthisak P. (2009), A simple isocratic HPLC method for the simultaneous determination of curcuminoids in commercial turmeric extracts, Phytochemical Analysis, 20(4), pp. 314-319. 
3. Jayaprakasha G.K., Rao L.J., Sakariah K.K. (2006), Antioxidant activities of curcumin, demethoxycurcumin and bisdemethoxycurcumin, Food Chemistry, 98(4), pp. 720-724.

4. Kant V., Gopal A., Pathak N.N., Kumar P., Tandan S.K., Kumar D. (2014), Antioxidant and anti-inflammatory potential of curcumin accelerated the cutaneous wound healing in streptozotocin-induced diabetic rats, International Immunopharmacology, 20(2), pp. 322-330.

5. Somchit M., Changtam C., Kimseng R., Utaipan T., Lertcanawanichakul M., Suksamrarn A., Chunglok W. (2014), Demethoxycurcumin from Curcuma longa rhizome suppresses iNOS induction in an in vitro inflamed human intestinal mucosa model, Asian Pacific Journal of Cancer Prevention, 15(4), pp. 1807-1810.

6. Aggarwal B.B., Kumar A., Bharti A.C. (2003), Anticancer potential of curcumin: Preclinical and clinical studies, Anticancer Research, 23(1A), pp. 363-398.

7. Ko Y.C., Hsu S.C., Liu H.C., Hsiao Y.T., Hsia T.C., Yang S.T., Hsu W.H., Chung J.G. (2015), Demethoxycurcumin alters gene expression associated with DNA damage, cell cycle and apoptosis in human lung cancer NCI-H460 cells in vitro, In Vivo, 29(1), pp. 83-94.

8. Panahi Y., Badeli R., Karami G.R., Sahebkar A. (2015), Investigation of the efficacy of adjunctive therapy with bioavailability-boosted curcuminoids in major depressive disorder, Phytotherapy Research, 29(1), pp. 17-21.

9. Shao Y., Zhu W., Da J., Xu M., Wang Y., Zhou J., Wang Z. (2017), Bisdemethoxycurcumin in combination with $\alpha$-PD-L1 antibody boosts immune response against bladder cancer, OncoTargets and Therapy, 10, pp. 2675-2683.

10. Ahmed T., Gilani A.H. (2014), Therapeutic potential of turmeric in Alzheimer's disease: curcumin or curcuminoids, Phytotherapy Research, 28(4), pp. 517-525.

11. Price L.C., Buescher R.W. (1997), Kinetics of alkaline degradation of the food pigments curcumin and curcuminoids, Journal of Food Science, 62(2), pp. 267-269.

12. (2004), Curcumin, Chemical and Technical Assessment, 61st JECFA. pp. 1-8. Available at: ftp://ftp.fao.org/es/esn/jecfa/cta/CTA_61_Curcumin.pdf.

13. Kalsi P.S. (2006), Chapter 2: Delocalized Chemical Bonding, Organic Reactions and Their Mechanisms (Second Edition), New Delhi, India: New Age International (P) Limited, pp. 37-80.

14. Rege S.A., Momin S.A., Wadekar S.D., Bhowmick D.N., Pratap A.P. (2014), Effect of demethoxycurcumin and bisdemethoxycurcumin on antioxidant activity of curcumin in refined sunflower oil, Journal of Food Processing and Preservation, 38(1), pp. 296303.

15. Antonov L., Deneva V., Simeonov S., Kurteva V., Crochet A., Fromm K.M., Shivachev B., Nikolova R., Savarese M., Adamo C. (2015), Controlled tautomeric switching in azonaphthols tuned by substituents on the phenyl ring, ChemPhysChem, 16(3), pp. 649-657.

16. Tonnesen H.H., Karlsen J. (1985), Studies of curcumin and curcuminoids: V. Alkaline degradation of curcumin, Zeitschrift für Lebensmittel-Untersuchung und-Forschung, 180(2), pp. 132-134.

17. Rege S.A., Momin S.A., Bhowmick D.N., Pratap A.P. (2012), Stabilization of emulsion and butter like products containing essential fatty acids using kalonji seeds extract and curcuminoids, Journal of Oleo Science, 61(1), pp. 11-16.

18. Priyadarsini K.I. (2013), Chemical and structural features influencing the biological activity of curcumin, Current Pharmaceutical Design, 19(11), pp. 2093-2100.

19. Rege S.A., Arya M., Momin S.A. (2019), Structure activity relationship of tautomers of curcumin: a review, Ukrainian Food Journal, 8(1), pp. 45-60. 